\title{
On the Kirwan map for moduli of Higgs bundles
}

\author{
Emily Cliff, Thomas Nevins ${ }^{\dagger}$ and Shiyu Shen
}

\begin{abstract}
Let $C$ be a smooth complex projective curve and $G$ a connected complex reductive group. We prove that if the center $Z(G)$ of $G$ is disconnected, then the Kirwan map $H^{*}(\operatorname{Bun}(G, C), \mathbb{Q}) \rightarrow H^{*}\left(\mathcal{M}_{\text {Higgs }}^{\text {ss }}(G, C), \mathbb{Q}\right)$, from the cohomology of the moduli stack of all $G$-bundles to the moduli stack of semistable $G$-Higgs bundles, cannot be surjective. We establish similar results for intersection cohomology and for the cohomology of the coarse moduli spaces. The proof uses a Borel-Quillen-style localization result for equivariant cohomology of stacks to reduce to an explicit calculation.
\end{abstract}

\section{Introduction}

Let $C$ be a nonsingular complex projective curve of genus $g \geqslant 2$. Let $G$ be a connected complex reductive group. Associated with $C$ and $G$, there are

- a moduli stack $\mathcal{M}_{\mathrm{Higgs}}^{\mathrm{ss}}(G, C)$ of semistable $G$-Higgs bundles on $C$,

- a coarse moduli space $M_{\mathrm{Higgs}}^{\mathrm{ss}}(G, C)$ of semistable $G$-Higgs bundles on $C$, and

- a morphism $\mathcal{M}_{\mathrm{Higgs}}^{\mathrm{ss}}(G, C) \stackrel{p}{\rightarrow} M_{\mathrm{Higgs}}^{\mathrm{ss}}(G, C)$.

It is important to compute the cohomology ring $H^{*}\left(\mathcal{M}_{\text {Higgs }}^{\text {ss }}(G, C)\right)$ in the topological analogue of the geometric Langlands program and, relatedly, in supersymmetric gauge theory, where the quantum cohomology ring (a deformation of the cohomology ring itself) is the chiral ring of a $2 D$ $\sigma$-model associated with a topologically twisted $4 D$ gauge theory (cf. [KW07]). Note that here and throughout, all cohomology is taken with $\mathbb{Q}$-coefficients. The ring $H^{*}\left(\mathcal{M}_{\mathrm{Higgs}}^{\mathrm{ss}}(G, C)\right)$ accepts a natural map, the "Kirwan map," from a tensor product of symmetric and exterior algebras, and it is natural to hope to use this as the starting point for a presentation of $H^{*}\left(\mathcal{M}_{\text {Higgs }}^{\text {ss }}(G, C)\right)$.

The main result of the present paper is that the above hope fails: the Kirwan map is never surjective when the gauge group $G$ has disconnected center.

\subsection{Kirwan map for $\mathcal{M}_{\text {Higgs }}^{\text {ss }}(G, C)$}

The connected components of the moduli stacks $\operatorname{Bun}(G, C)$ and $\mathcal{M}_{\text {Higgs }}^{\text {ss }}(G, C)$ and the moduli space $M_{\text {Higgs }}^{\text {ss }}(G, C)$ are indexed by the abelian group $\pi_{1}(G)$ (cf. [GO17] for the latter). Let $Z=Z(G)$ denote the center of $G$. The group $Z$ splits as a product, $Z \cong Z^{\circ} \times \pi_{0}(Z)$, where

Received 28 November 2019, accepted in final form 12 November 2020.

2020 Mathematics Subject Classification 14D20, $14 \mathrm{D} 23$.

Keywords: semistable $G$-Higgs bundles, Kirwan map, Quillen localization.

This journal is (C) Foundation Compositio Mathematica 2021. This article is distributed with Open Access under the terms of the Creative Commons Attribution Non-Commercial License, which permits non-commercial reuse, distribution, and reproduction in any medium, provided that the original work is properly cited. For commercial re-use, please contact the Foundation Compositio Mathematica.

$\dagger$ Thomas Nevins passed away while this paper was under review. The first and third authors are grateful for his guidance and mentorship during their post-doc and $\mathrm{PhD}$, respectively. They miss him. 


\section{E. Cliff, T. Nevins And S. Shen}

the identity component of $Z$ is $Z^{\circ}$ and the component group is $\pi_{0}(Z)$. The abelian group stack $\operatorname{Bun}(Z, C)$, the moduli stack of principal $Z$-bundles, acts on $\mathcal{M}_{\text {Higgs }}^{\text {ss }}(G, C)$, inducing an action of the coarse moduli space $\underline{\operatorname{Bun}}(Z, C)$ on $M_{\text {Higgs }}^{\text {ss }}(G, C)$. The group $\pi_{1}(G)$ contains a finite-index subgroup, the image of $\iota: \pi_{1}\left(Z^{\circ}\right) \rightarrow \pi_{1}(G)$ (cf. (3.6) and Proposition 3.8).

For $\eta \in \pi_{1}(G)$, write $\operatorname{Bun}(G, C)_{\eta}, \mathcal{M}_{\text {Higgs }}^{\text {ss }}(G, C)_{\eta}, M_{\text {Higgs }}^{\text {ss }}(G, C)_{\eta}$ for the corresponding components. When we apply singular cohomology with $\mathbb{Q}$-coefficients, the maps

$$
\operatorname{Bun}(G, C)_{\eta}<{ }^{k_{\eta}} \mathcal{M}_{\mathrm{Higgs}}^{\mathrm{ss}}(G, C)_{\eta} \stackrel{p_{\eta}}{\longrightarrow} M_{\mathrm{Higgs}}^{\mathrm{ss}}(G, C)_{\eta}
$$

induce a diagram

$$
H^{*}\left(\operatorname{Bun}(G, C)_{\eta}\right) \stackrel{\kappa_{\eta}=k_{\eta}^{*}}{\longrightarrow} H^{*}\left(\mathcal{M}_{\mathrm{Higgs}}^{\mathrm{ss}}(G, C)_{\eta}\right) \stackrel{p_{\eta}^{*}}{\longleftarrow} H^{*}\left(M_{\mathrm{Higgs}}^{\mathrm{ss}}(G, C)_{\eta}\right) .
$$

Here for a semistable $G$-Higgs bundle $(P, \theta)$, we have $k_{\eta}(P, \theta)=P$. The left-hand map $\kappa_{\eta}$ is the (hyperkähler) Kirwan map for the moduli stack of semistable $G$-Higgs bundles. Only when $G=\mathrm{SL}_{2}, \mathrm{GL}_{2}$, or $\mathrm{PGL}_{2}$ is it known for all $\eta$ whether $\kappa_{\eta}$ is surjective.

Remark 1.1. The stack $\mathcal{M}_{\text {Higgs }}^{\text {ss }}(G, C)_{\eta}$ is obtained, essentially, by hyperkähler reduction of an infinite-dimensional affine space by the action of the gauge group $\mathcal{G}=\operatorname{Maps}_{\eta}\left(C, G^{\mathrm{cpt}}\right)$, a twisted version of $C^{\infty}$-maps to the compact real form $G^{\mathrm{cpt}}$ of $G$. The classifying space $B \operatorname{Maps}_{\eta}\left(C, G^{\mathrm{cpt}}\right)$ is, moreover, homotopy equivalent to $\operatorname{Bun}(G, C)_{\eta}$, and hence the map $\kappa_{\eta}$ is indeed (up to homotopy) the hyperkähler Kirwan map associated with this hyperkähler reduction.

\subsection{Main theorem}

As we explain in Section 3, the action of $\operatorname{Bun}(Z, C)$ on $\mathcal{M}_{\text {Higgs }}^{\text {ss }}(G, C)$ induces compatible actions of the finite group ${ }^{1} \operatorname{Bun}\left(\pi_{0}(Z), C\right)$ on $H^{*}(\operatorname{Bun}(G, C))$, where it is trivial, as well as on $H^{*}\left(\mathcal{M}_{\text {Higgs }}^{\text {ss }}(G, C)\right)$ and $H^{*}\left(M_{\text {Higgs }}^{\text {ss }}(G, C)\right)$. In Definition 7.1 , we identify a particular $G$-bundle, denoted by $P_{0}$, the image of 0 in the Hitchin base under the Hitchin section; write $\mathcal{M}_{\text {Higgs }}^{\text {ss }}(G, C)_{\eta_{0}}$ for the component of $\mathcal{M}_{\text {Higgs }}^{\text {ss }}(G, C)$, indexed by the element $\eta_{0} \in \pi_{1}(G)$ in which $P_{0}$ lies. We prove the following result.

Theorem 1.2. If the component group $\pi_{0}(Z(G))$ is nontrivial, then for every $\eta \in \eta_{0}+\pi_{1}\left(Z^{\circ}\right)$, the following hold:

(1) The cohomology groups $H^{*}\left(\mathcal{M}_{\text {Higgs }}^{\text {ss }}(G, C)_{\eta}\right)$ and $H^{*}\left(M_{\text {Higgs }}^{\text {ss }}(G, C)_{\eta}\right)$ contain the regular representation $\mathbb{Q}\left[\operatorname{Bun}\left(\pi_{0}(Z), C\right)\right]$ of $\operatorname{Bun}\left(\pi_{0}(Z), C\right)$.

(2) Similarly, the intersection cohomology groups $I H^{*}\left(\mathcal{M}_{\mathrm{Higgs}}^{\mathrm{ss}}(G, C)_{\eta}\right), I H^{*}\left(M_{\mathrm{Higgs}}^{\mathrm{ss}}(G, C)_{\eta}\right)$ contain the regular representation $\mathbb{Q}\left[\operatorname{Bun}\left(\pi_{0}(Z), C\right)\right]$ of $\operatorname{Bun}\left(\pi_{0}(Z), C\right)$.

(3) In particular, the Kirwan map $\kappa_{\eta}$ fails to be surjective.

(4) We have $p_{\eta}^{*} H^{*}\left(M_{\text {Higgs }}^{\text {ss }}(G, C)_{\eta}\right) \nsubseteq \kappa_{\eta}^{*} H^{*}\left(\operatorname{Bun}(G, C)_{\eta}\right)$.

For semisimple $G$, Theorem 1.2 says that the Kirwan map for $\mathcal{M}_{\mathrm{Higgs}}^{\mathrm{ss}}(G, C)$ can only be surjective when $G$ is of adjoint type, (roughly) generalizing the work of Hitchin (cf. [Hit87] and [HT03]) for rank $n$ bundles of degree 1 and fixed determinant (a variant of $\mathrm{SL}_{n}$-bundles) and [DWWW11] for $G=\mathrm{SL}_{2}$.

\footnotetext{
${ }^{1}$ The stack $\operatorname{Bun}\left(\pi_{0}(Z), C\right)$ is a gerbe over a finite group, but the action on $H^{*}$ factors through the finite group.
} 


\section{ON THE KIRWAN MAP FOR MODULI OF HiggS BUNDLES}

Remark 1.3. The proof shows (see Remark 7.8) that the representation $\mathbb{Q}\left[\operatorname{Bun}\left(\pi_{0}(Z), C\right)\right]$ of $\operatorname{Bun}\left(\pi_{0}(Z), C\right)$ in part (1) of Theorem 1.2 appears in the pure parts

$$
\bigoplus_{k} \operatorname{Gr}_{k}^{W}\left(H^{k}\left(\mathcal{M}_{\mathrm{Higgs}}^{\mathrm{ss}}(G, C)_{\eta}\right)\right) \text { and } \bigoplus_{k} \operatorname{Gr}_{k}^{W}\left(H^{*}\left(M_{\mathrm{Higgs}}^{\mathrm{ss}}(G, C)_{\eta}\right)\right)
$$

with respect to the mixed Hodge structure. In particular, the Kirwan map (whose domain $H^{*}\left(\operatorname{Bun}(G, C)_{\eta}\right)$ is pure $)$ fails to surject even onto the pure part of the cohomology.

\subsection{Kähler versus hyperkähler Kirwan map}

In the case of (algebro-geometric) Kähler quotients (or, equivalently, GIT quotients) of smooth varieties (either projective, or quasiprojective under some additional assumptions) rather than hyperkähler quotients, the Kirwan map is known to be surjective; see [Kir84] for the canonical result in a comprehensive treatment. This provides an essential starting point (explicit generators) for the computation of the cohomology of Kähler/GIT quotients.

By contrast, the Kirwan map for hyperkähler quotients like the ones we treat here is considerably more subtle - thus, so is the computation of the cohomology of such quotients. ${ }^{2}$ The hyperkähler Kirwan map is known to be surjective for hypertoric varieties, quiver varieties, and a limited number of other cases [JKK09, Mar02, MN18, DWWW11], thus providing polynomial rings surjecting onto the cohomology rings of such varieties. In the case of $\mathcal{M}_{\text {Higgs }}^{\text {ss }}(G, C)$, the cohomology $H^{*}(\operatorname{Bun}(G, C))$ is known to be a free graded-commutative $\mathbb{Q}$-algebra, so if the hyperkähler Kirwan map $\kappa_{\eta}$ for $\mathcal{M}_{\text {Higgs }}^{\text {ss }}(G, C)$ were surjective, it would express the cohomology of $\mathcal{M}_{\text {Higgs }}^{\text {ss }}(G, C)$ as a quotient of a free graded-commutative algebra. And indeed, for $G=\mathrm{GL}_{n}$ and coprime rank $n$ and degree $d$ (where the map $p$ becomes a $\mathbb{G}_{m}$-gerbe over a smooth variety), it is proven in [Mar02] that $\kappa_{\eta}$ is surjective, as it also is for $G=\mathrm{GL}_{2}, \mathrm{PGL}_{2}$ and $d=0$ in [DWWW11].

By contrast, Theorem 1.2 shows that $\kappa$ is not surjective for $\mathcal{M}_{\text {Higgs }}^{\text {ss }}(G, C)$ whenever $Z(G)$ is disconnected. More generally, experts seem to believe that there should be many examples of hyperkähler reductions for which the hyperkähler Kirwan map fails to be surjective, though there seem to be (essentially) no documented examples outside of $\mathcal{M}_{\mathrm{Higgs}}^{\mathrm{ss}}(G, C)$ : the paper [MN19] provides other explicit examples of finite-dimensional hyperkähler manifolds $\mathrm{M}$ with the action of compact groups $K$ for which the hyperkähler Kirwan map $H_{K}^{*}(\mathrm{M}) \rightarrow H^{*}(\mathrm{M} / / / K)$ fails to be surjective, but the construction in [MN19] is also inspired by the case of $G$-Higgs bundles.

We hope that the growing lists of examples in which the hyperkähler Kirwan map is [JKK09, Mar02, MN18, DWWW11] or is not ([DWWW11, HT03, MN19] and the present paper) surjective will eventually provide enough data to indicate a characterization of when $\kappa$ should be surjective and how surjectivity can fail - and thus indicate how to calculate the cohomology of hyperkähler quotients.

\subsection{Strategy of proof}

The proof of Theorem 1.2 makes use of the commuting actions of the multiplicative group $\mathbb{G}_{m}$, acting by scaling the Higgs field, and the abelian group stack $\operatorname{Bun}(Z, C)$ of principal $Z$-bundles, acting by "tensoring" the $G$-Higgs bundle, on $\mathcal{M}_{\text {Higgs }}^{\text {ss }}(G, C)$ and $M_{\text {Higgs }}^{\text {ss }}(G, C)$. Section 3 develops basic properties of the action of $\operatorname{Bun}(Z, C)$, in particular showing that $\operatorname{Bun}(Z, C)$ acts infinitesi-

\footnotetext{
${ }^{2}$ Informally, in the case of Kähler quotients one uses Morse theory on a smooth variety, whereas in the case of hyperkähler quotients, one needs a Morse-theoretic analysis of a singular variety - the zero preimage of a complex moment map - which has proven to be intractable in most cases thus far.
} 


\section{E. Cliff, T. Nevins and S. Shen}

mally freely on $\mathcal{M}_{\text {Higgs }}^{\text {ss }}(G, C)$ and that the induced action of $H^{1}\left(C, \pi_{0}(Z)\right)$, the group of isomorphism classes of principal $\pi_{0}(Z)$-bundles, preserves connected components of $\mathcal{M}_{\text {Higgs }}^{\text {ss }}(G, C)$.

We show that whenever $H^{1}\left(C, \pi_{0}(Z)\right)$ is nontrivial, it acts nontrivially on $H^{*}\left(\mathcal{M}_{\mathrm{Higgs}}^{\text {ss }}(G, C)_{\eta}\right)$ and $H^{*}\left(M_{\mathrm{Higgs}}^{\mathrm{ss}}(G, C)_{\eta}\right)$, whereas it acts trivially on $H^{*}\left(\operatorname{Bun}(G, C)_{\eta}\right)$. Essentially the same idea was used in [DWWW11] (without using the $\mathbb{G}_{m}$-action to simplify) and [HT03].

Section 4 develops the principal computational tool in our proof, a Borel-Quillen-style localization theorem for equivariant cohomology of stacks that, applied to the $\mathbb{G}_{m}$-action, allows us to reduce to a calculation on a certain $\mathbb{G}_{m}$-fixed locus, $\mathcal{M}_{\text {Higgs }}^{\text {ss }}(G, C)_{\text {fixed }}$ in the notation of that section. We show by explicit construction that $\mathcal{M}_{\text {Higgs }}^{\text {ss }}(G, C)_{\text {fixed }}$ contains a union of connected components isomorphic to $\operatorname{Bun}(Z, C)$, compatibly with the action of $\operatorname{Bun}(Z, C)$ on $\mathcal{M}_{\mathrm{Higgs}}^{\mathrm{ss}}(G, C)$. It follows that $H^{1}\left(C, \pi_{0}(Z)\right)$ acts nontrivially on $H^{*}\left(\mathcal{M}_{\text {Higgs }}^{\text {ss }}(G, C)\right)$, whereas it acts trivially on $H^{*}(\operatorname{Bun}(G, C))$. This proves the first assertion of Theorem 1.2 for the cohomology of the stack (and essentially the same proof gives the intersection cohomology statement); the proof for the moduli space is similar.

Although the use of $\mathbb{C}^{*}$-localization to establish topological features of algebraic varieties or other topological spaces is completely standard, it seems to be new to use it for Artin stacks rather than merely Deligne-Mumford stacks. While the statement we use is not hard, it appears to be new. It turns out that there is a broader story of such localizations that seems not to be as well known as we think warranted, and that we plan to develop further in a future paper.

We also note that we have chosen to include some basic facts that could be cited in the literature rather than developed from scratch as we have done here - while this runs the risk of giving the impression that we claim originality for facts well known to the community, we simply note that even the present more-or-less self-contained paper is not long.

\section{Notation, conventions, and basics of $G$-Higgs bundles}

\subsection{Notation and conventions}

Throughout the paper, we work with varieties, schemes, and stacks over $\mathbb{C}$. We write $\mathbb{G}_{m}$ for the multiplicative group $\mathbb{C}^{*}$. We write $\mathbb{A}^{1}(d)$ for $\mathbb{A}^{1}$ with the $\mathbb{G}_{m}$-action of weight $d$, that is, $t \cdot v=t^{d} v$.

As in the introduction, all singular cohomology groups are taken with $\mathbb{Q}$-coefficients.

Throughout the paper, we fix a smooth projective (connected) complex curve $C$ of genus $g \geqslant 2$. Choose a spin bundle $K_{C}^{1 / 2}$, and let $\mathrm{K}^{1 / 2}$ denote the associated $\mathbb{G}_{m}$-bundle, so that $K_{C}^{1 / 2}=\mathrm{K}^{1 / 2} \times_{\mathbb{G}_{m}} \mathbb{A}^{1}(1)$. Let $\mathrm{K}^{-1 / 2}$ denote the dual $\mathbb{G}_{m}$-bundle, so $\mathrm{K}^{-1 / 2} \times_{\mathbb{G}_{m}} \mathbb{A}^{1}(-1) \cong K_{C}^{1 / 2}$.

We also work with a fixed connected, reductive complex algebraic group $G$ with center $Z=Z(G)$. We fix a Borel subgroup $B \subset G$ and a maximal torus $T \subset B$; let $U \subset B$ denote the unipotent radical. We write $\mathfrak{g}=\operatorname{Lie}(G), \mathfrak{b}=\operatorname{Lie}(B), \mathfrak{n}=\operatorname{Lie}(U), \mathfrak{z}=\operatorname{Lie}(Z)$, and $\mathcal{N}$ for the nilpotent cone of $\mathfrak{g}$. We let $\mathfrak{g}^{\text {reg }}$ denote the regular locus of $\mathfrak{g}$.

For an affine algebraic group $H$, we write $\operatorname{Bun}(H, C)$ for the moduli stack of principal $H$ bundles on $C$. When the identity component of $H$ is an algebraic torus, we write $\operatorname{Bun}(H, C)$ for the coarse moduli scheme of principal $H$-bundles. We use the terminology "abelian group" and "commutative group" interchangeably. However, we prefer "commutative group scheme/stack" because of the risk that "abelian group scheme" suggests that the scheme is proper (an abelian variety) over some base. 


\section{On the KiRWAN MAP FOR MOdUli OF Higgs BUndLES}

\subsection{Basics on $\mathcal{M}_{\mathrm{Higgs}}^{\mathrm{ss}}(G, C)$ and $M_{\mathrm{Higgs}}^{\mathrm{ss}}(G, C)$}

Let $(\mathcal{E}, \theta)$ be a $G$-Higgs bundle on $C$. Recall that for a parabolic subgroup $\mathrm{P} \subset G$ and P-reduction $\mathcal{E}_{\mathrm{P}}$ of $\mathcal{E}$, we say that $\mathcal{E}_{\mathrm{P}}$ is compatible with $\theta$ if $\theta$ is (the image of) a section of $\mathcal{E}_{\mathrm{P}} \times_{\mathrm{P}} \mathfrak{p} \otimes \Omega_{C}^{1} \subset$ $\mathcal{E}_{\mathrm{P}} \times \mathrm{P} \mathfrak{g} \otimes \Omega_{C}^{1}$. Then the $G$-Higgs bundle $(\mathcal{E}, \theta)$ is semistable, respectively stable, if for every proper parabolic subgroup $\mathrm{P} \subset G$ and P-reduction $\mathcal{E}_{\mathrm{P}}$ of $\mathcal{E}$ compatible with $\theta$, the bundle $\mathcal{E}_{\mathrm{P}} \times \mathrm{P} \mathfrak{g} / \mathfrak{p}$ has nonpositive, respectively negative, degree. The center $Z(G)$ always acts by automorphisms of the pair $(\mathcal{E}, \theta)$; we say that $(\mathcal{E}, \theta)$ is simple if $\operatorname{Aut}(\mathcal{E}, \theta)=Z(G)$.

Notation 2.1. Let $\mathcal{M}_{\text {Higgs }}^{\text {ss }}(G, C)$ denote the moduli stack of semistable $G$-Higgs bundles and $M_{\text {Higgs }}^{\text {ss }}(G, C)$ the coarse moduli space of semistable $G$-Higgs bundles.

The quasiprojective scheme $M_{\text {Higgs }}^{\text {ss }}(G, C)$ is a coarse space of $\mathcal{M}_{\text {Higgs }}^{\text {ss }}(G, C)$. We note some well-known facts (cf. the "Main Properties" of [Alp13]).

Proposition 2.2. Consider the morphism $p: \mathcal{M}_{\mathrm{Higgs}}^{\mathrm{ss}}(G, C) \rightarrow M_{\mathrm{Higgs}}^{\mathrm{ss}}(G, C)$.

(1) The morphism $p: \mathcal{M}_{\mathrm{Higgs}}^{\mathrm{ss}}(G, C) \rightarrow M_{\mathrm{Higgs}}^{\mathrm{ss}}(G, C)$ is surjective.

(2) Each fiber of $p$ contains a unique closed point of the moduli stack.

(3) The morphism $p$ is universally closed; in particular, the topology of $M_{\mathrm{Higgs}}^{\mathrm{ss}}(G, C)$ is the quotient topology of the stack $\mathcal{M}_{\mathrm{Higgs}}^{\text {ss }}(G, C)$.

The conditions of stability, respectively simplicity, define open substacks $\mathcal{M}_{\text {Higgs }}^{\mathrm{s}}(G, C)$, respectively $\mathcal{M}_{\text {Higgs }}^{\text {spl }}(G, C)$ of $\mathcal{M}_{\text {Higgs }}^{\text {ss }}(G, C)$. Write $\mathcal{M}_{\text {Higgs }}^{\text {st-spl }}(G, C):=\mathcal{M}_{\text {Higgs }}^{\text {s }}(G, C) \cap \mathcal{M}_{\text {Higgs }}^{\text {spl }}(G, C)$.

For the morphism $p$ of Proposition 2.2, we have

$$
p^{-1}\left(p\left(\mathcal{M}_{\mathrm{Higgs}}^{\mathrm{s}}(G, C)\right)\right)=\mathcal{M}_{\mathrm{Higgs}}^{\mathrm{s}}(G, C), \quad p^{-1}\left(p\left(\mathcal{M}_{\mathrm{Higgs}}^{\mathrm{st}-\mathrm{spl}}(G, C)\right)\right)=\mathcal{M}_{\mathrm{Higgs}}^{\mathrm{st}-\mathrm{spl}}(G, C) .
$$

Notation 2.3. We write

$$
M_{\mathrm{Higgs}}^{\mathrm{s}}(G, C):=p\left(\mathcal{M}_{\mathrm{Higgs}}^{\mathrm{s}}(G, C)\right) \quad \text { and } \quad M_{\mathrm{Higgs}}^{\mathrm{st-spl}}(G, C):=p\left(\mathcal{M}_{\mathrm{Higgs}}^{\mathrm{st-spl}}(G, C)\right) .
$$

Lemma 2.4. The morphism $\mathcal{M}_{\text {Higgs }}^{\text {st-spl }}(G, C) \rightarrow M_{\text {Higgs }}^{\text {st-spl }}(G, C)$ is a torsor for the commutative group stack $B Z$, inducing an identification $M_{\text {Higgs }}^{\text {st-spl }}(G, C)=\mathcal{M}_{\text {Higgs }}^{\text {st-spl }}(G, C) / B Z$.

Consider the product $C \times \operatorname{Bun}(G, C) \rightarrow B G \times B \mathbb{G}_{m}$ of the classifying morphism $C \times$ $\operatorname{Bun}(G, C) \rightarrow B G$ for the universal $G$-bundle and the composite $C \times \operatorname{Bun}(G, C) \rightarrow C \rightarrow B \mathbb{G}_{m}$, where the latter map classifies the $\mathbb{G}_{m}$-bundle associated with $\Omega_{C}^{1}$. The universal $G$-Higgs field then determines a morphism $C \times \mathcal{M}_{\text {Higgs }}^{\text {ss }}(G, C) \rightarrow \mathfrak{g} /\left(G \times \mathbb{G}_{m}\right)$ making the following square commute:

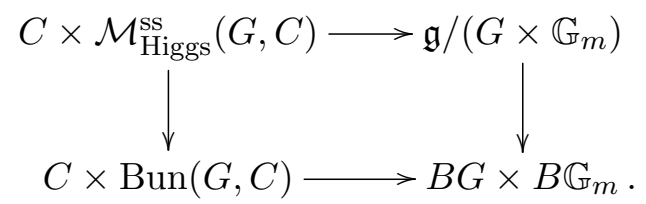

If $S \subset \mathfrak{g}$ is $\left(G \times \mathbb{G}_{m}\right)$-stable, we slightly abuse terminology by saying that $(P, \theta)$ takes values in $S /\left(G \times \mathbb{G}_{m}\right)$ if the restricted universal morphism $C \times\{(P, \theta)\} \rightarrow \mathfrak{g} /\left(G \times \mathbb{G}_{m}\right)$ does. A $G$-Higgs pair $(P, \theta)$ is regular if it takes values in $\mathfrak{g}^{\text {reg }} /\left(G \times \mathbb{G}_{m}\right)$ and nilpotent if it takes values in $\mathcal{N} /\left(G \times \mathbb{G}_{m}\right)$. The following is immediate from the properness of $C$.

Lemma 2.5. The regular $G$-Higgs bundles form an open substack of $\mathcal{M}_{\mathrm{Higgs}}^{\mathrm{ss}}(G, C)$, denoted by $\mathcal{M}_{\text {Higgs }}^{\text {ss }}(G, C)_{\text {reg. }}$ 


\section{E. Cliff, T. Nevins and S. Shen}

\section{Principal $Z(G)$-bundles and the action on $G$-Higgs bundles}

\subsection{Principal $Z(G)$-bundles}

Write $Z=Z(G)$. We have a short exact sequence

$$
1 \rightarrow Z^{\circ} \rightarrow Z \rightarrow \pi_{0}(Z) \rightarrow 1,
$$

where $Z^{\circ}$ is the identity component. Note that $Z^{\circ}$ is a torus and $\pi_{0}(Z)$ is a finite abelian group.

Lemma 3.1. (1) Suppose that $1 \longrightarrow D \longrightarrow A \longrightarrow F \longrightarrow 1$ is an exact sequence of abelian groups, where $F$ is finitely generated abelian and $D$ is a divisible group. Then $A \cong F \times D$.

(2) In particular, for a connected reductive group $G$ over $\mathbb{C}$, we have $Z(G) \cong Z^{\circ} \times \pi_{0}(Z)$.

Remark 3.2. In light of Lemma 3.1(2), we henceforth fix a choice of splitting $Z(G) \cong Z^{\circ} \times \pi_{0}(Z)$.

For a commutative group scheme $A$ whose identity component is an algebraic torus, write $\underline{\operatorname{Bun}}(A, C)=H^{1}(C, A)$ for the coarse moduli space of $A$-bundles on $C$. The splitting $Z(G) \cong$ $Z^{\circ} \times \pi_{0}(Z)$ determines an isomorphism of group schemes

$$
\underline{\operatorname{Bun}}(Z, C)=\underline{\operatorname{Bun}}\left(Z^{\circ}, C\right) \times \underline{\operatorname{Bun}}\left(\pi_{0}(Z), C\right) .
$$

We have $\operatorname{Bun}(A, C) \cong B(A) \times \underline{\operatorname{Bun}}(A, C)$ when $A$ is any of the groups $Z^{\circ}, Z$, or $\pi_{0}(Z)$; in particular, $\operatorname{Bun}\left(\pi_{0}(Z), C\right)$ is a gerbe over a finite abelian group scheme. Since $Z^{\circ}$ is a torus,

$$
\underline{\operatorname{Bun}}\left(Z^{\circ}, C\right) \cong \operatorname{Jac}(C)^{r} \times \mathbb{Z}^{r} .
$$

Proposition 3.3. We have

$$
\operatorname{Bun}(Z, C) \cong B\left(Z^{\circ}\right) \times B\left(\pi_{0}(Z)\right) \times \operatorname{Jac}(C)^{r} \times \pi_{0}\left(\underline{\operatorname{Bun}}\left(Z^{\circ}, C\right)\right) \times \pi_{0}\left(\underline{\operatorname{Bun}}\left(\pi_{0}(Z), C\right)\right) .
$$

In particular, $\operatorname{Bun}(Z, C)$ is a disjoint union of connected components, each of which is a BZ-torsor over a nonsingular projective variety.

\subsection{Action of $\operatorname{Bun}(Z, C)$ on $\operatorname{Bun}(G, C)$ and $T^{*} \operatorname{Bun}(G, C)$}

The stack $\operatorname{Bun}(Z, C)$ of principal $Z$-bundles on $C$ is a commutative group stack, with operation $(\mathcal{E}, \mathcal{F}) \mapsto \mathcal{E} \times{ }_{Z} \mathcal{F}$. The group stack $\operatorname{Bun}(Z, C)$ acts naturally on $\operatorname{Bun}(H, C)$ as well, by the same formula, when $\mathcal{F}$ is a principal $H$-bundle for any closed subgroup $Z \subseteq H \subseteq G$. In particular, the action of $\operatorname{Bun}(Z, C)$ on $\operatorname{Bun}(G, C)$ induces an action of $\operatorname{Bun}(Z, C)$ on $T^{*} \operatorname{Bun}(G, C)$, the moduli stack of all $G$-Higgs bundles on $C$.

Remark 3.4. The action of $\operatorname{Bun}(Z, C)$ preserves parabolic reductions of a given $G$-bundle, and $Z$ acts trivially on $\mathfrak{g} / \mathfrak{p}$ for any parabolic subalgebra $\mathfrak{p} \subset \mathfrak{g}$. Hence, the action of $\operatorname{Bun}(Z, C)$ on $T^{*} \operatorname{Bun}(G, C)$ preserves the open substacks of semistable, stable, and simple $G$-Higgs bundles (definitions as in Section 2.2).

Let $(P, \theta)$ be a $G$-Higgs bundle. One gets a complex of vector bundles over $C$,

$$
\operatorname{Def}(P, \theta)=P \times_{G} \mathfrak{g} \stackrel{[\theta,-]}{\longrightarrow} P \times_{G} \mathfrak{g} \otimes \Omega_{C}^{1}
$$

with $P \times_{G} \mathfrak{g}$ in cohomological degree -1 . For the fiber of the tangent complex at $(P, \theta)$ one has

$$
\mathbb{T}_{(P, \theta)}\left(T^{*} \operatorname{Bun}(G, C)\right) \simeq \check{C}^{\bullet}(C, \operatorname{Def}(P, \theta)),
$$

where $\check{C} \bullet$ denotes the $\check{C}$ ech complex associated with any choice of affine open cover of $C$. 


\section{On the KiRWAN MAP FOR MODUli OF Higgs BUndLES}

Note that the Lie algebra $\mathfrak{g}$ splits as a $G$-representation, $\mathfrak{g} \cong \mathfrak{z} \oplus \mathfrak{g}_{\text {ad }}$. It follows that $P \times_{G} \mathfrak{z}[1]=$ $\mathcal{O}_{C} \otimes \mathfrak{z}[1]$ is a direct summand of $\operatorname{Def}(P, \theta)$. Writing triv for the trivial $Z$-bundle on $C$, we have $\mathbb{T}_{\text {triv }}(\operatorname{Bun}(Z, C)) \simeq \check{C}^{\bullet}\left(C, \mathcal{O}_{C} \otimes \mathfrak{z}[1]\right)$. We conclude as follows.

Lemma 3.5. For any choice of $G$-Higgs bundle $(P, \theta)$, the infinitesimal actions of $\operatorname{Bun}(Z, C)$ on $T^{*} \operatorname{Bun}(G, C)$ and $\operatorname{Bun}(G, C)$,

$$
\mathbb{T}_{\text {triv }}(\operatorname{Bun}(Z, C)) \rightarrow \mathbb{T}_{(P, \theta)}\left(T^{*} \operatorname{Bun}(G, C)\right) \rightarrow \mathbb{T}_{P}(\operatorname{Bun}(G, C)),
$$

express $\mathbb{T}_{\text {triv }}(\operatorname{Bun}(Z, C))$ as a direct summand of both $\mathbb{T}_{(P, \theta)}\left(T^{*} \operatorname{Bun}(G, C)\right)$ and $\mathbb{T}_{P}(\operatorname{Bun}(G, C))$.

Since $\operatorname{Bun}(Z, C)$ is a gerbe over a smooth scheme, we conclude as follows.

Corollary 3.6. For any choice of $G$-Higgs bundle $(P, \theta)$, the induced morphisms of "action on $P$, respectively $(P, \theta)$,"

$$
\operatorname{Bun}(G, C) \longleftarrow \operatorname{Bun}(Z, C) \longrightarrow T^{*} \operatorname{Bun}(G, C)
$$

are representable and injective on tangent spaces.

Proof. The homomorphisms $Z(G) \rightarrow \operatorname{Aut}(P, \theta)$ and $Z(G) \rightarrow \operatorname{Aut}(P)$ are always injective; hence, the morphisms are representable. By Lemma 3.5, the induced map on $H^{0}(\mathbb{T})$ is injective; hence, the morphisms are immersions.

\subsection{Connected components of $\operatorname{Bun}(G, C)$}

Let $\widetilde{G}$ denote the universal cover of $G$ as a complex-analytic Lie group, with the exact sequence

$$
1 \longrightarrow \pi_{1}(G) \longrightarrow \widetilde{G} \longrightarrow G \longrightarrow 1 \text {. }
$$

Each of these groups determines a complex-analytic sheaf of groups over the complex-analytic curve $C_{\text {an }}$, abusively denoted by the same symbol. The set of $\mathbb{C}$-points of the $\operatorname{stack} \operatorname{Bun}_{G}(C)$ is the (nonabelian) cohomology set $H^{1}\left(C_{\text {an }}, G\right)$. We recall (for example, from [Hof10]) that the set of connected components of the stack $\operatorname{Bun}_{G}(C)$ is classified by the connecting homomorphism of cohomology sets

$$
H^{1}\left(C_{\mathrm{an}}, G\right) \stackrel{\delta}{\rightarrow} H^{2}\left(C, \pi_{1}(G)\right)=\pi_{1}(G) ;
$$

that is, the connected components of $\operatorname{Bun}_{G}(C)$ are labeled by $\pi_{1}(G)$, and the fibers of $\delta$ are the isomorphism classes lying in the given connected component.

Via the splitting $Z=Z^{\circ} \times \pi_{0}(Z)$ (Remark 3.2), we get a homomorphism $\pi_{0}(Z) \hookrightarrow G$. Pulling back the exact sequence (3.4) gives an extension

$$
1 \longrightarrow \pi_{1}(G) \longrightarrow \widetilde{\pi_{0}(Z)} \longrightarrow \pi_{0}(Z) \longrightarrow 1
$$

inducing a commutative square

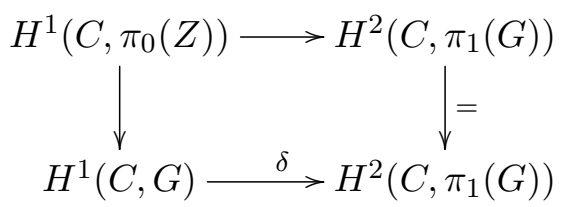

and making $H^{1}(C, G) \stackrel{\delta}{\rightarrow} H^{2}\left(C, \pi_{1}(G)\right)$ an equivariant map of $H^{1}\left(C, \pi_{0}(Z)\right)$-sets.

Proposition 3.7. The group $H^{1}\left(C, \pi_{0}(Z)\right)$ acts trivially on $H^{2}\left(C, \pi_{1}(G)\right)$. Thus, the abelian group stack $\operatorname{Bun}\left(\pi_{0}(Z), C\right)$ acts on $\operatorname{Bun}(G, C)$ preserving connected components. 


\section{E. Cliff, T. Nevins and S. Shen}

Proof. Via the commutative square, we see that it suffices to show that the boundary map $H^{1}\left(C, \pi_{0}(Z)\right) \rightarrow H^{2}\left(C, \pi_{1}(G)\right)$ associated with (3.5) is zero. But since the groups in (3.5) are discrete and $H^{*}(C, \mathbb{Z})$ is a free $\mathbb{Z}$-module, the universal coefficient theorem gives

$$
H^{1}\left(C, \widetilde{\pi_{0}(Z)}\right)=H^{1}(C, \mathbb{Z}) \otimes \widetilde{\pi_{0}(Z)} \rightarrow H^{1}(C, \mathbb{Z}) \otimes \pi_{0}(Z)=H^{1}\left(C, \pi_{0}(Z)\right),
$$

implying that the boundary map is zero.

\subsection{Action of $\operatorname{Bun}(Z, C)$ on components of $\operatorname{Bun}(G, C)$}

Now, choose a principal $G$-bundle $P$ on $C$, determining a $\mathbb{C}$-point of $\operatorname{Bun}(G, C)$. We obtain a morphism

$$
\psi_{P}: \operatorname{Bun}(Z, C) \rightarrow \operatorname{Bun}(G, C), \quad P_{Z} \mapsto P_{Z} \times_{Z} P .
$$

Proposition 3.8. The map of sets of connected components induced by the morphism $\psi_{P}$ maps $\pi_{0}\left(\operatorname{Bun}\left(Z^{\circ}, C\right)\right)=\pi_{1}\left(Z^{\circ}\right)$ injectively to $\pi_{0}(\operatorname{Bun}(G, C))=\pi_{1}(G)$. The image of $\pi_{0}\left(\operatorname{Bun}\left(Z^{\circ}, C\right)\right)$ $\rightarrow \pi_{0}(\operatorname{Bun}(G, C))$ is a finite-index subgroup.

Proof. It suffices to show that the homomorphism $H^{2}(\iota): H^{2}\left(C, \pi_{1}\left(Z^{\circ}\right)\right) \rightarrow H^{2}\left(C, \pi_{1}(G)\right)$ is injective with finite cokernel. But up to an isogeny, $G$ is the product of a torus $\mathbb{T}$ and a semisimple group, with the torus $\mathbb{T}$ thus being isogenous to $Z^{\circ}$. Since a semisimple group has finite $\pi_{1}$, we conclude that

$$
\text { the map } \quad \iota: \pi_{1}\left(Z^{\circ}\right) \rightarrow \pi_{1}(G) \text { has finite kernel and cokernel. }
$$

Since the domain of $\iota$ is torsion-free, the kernel is trivial. Finally, it follows from the universal coefficient theorem that $H^{2}(\iota)$ is obtained by tensoring $\pi_{1}\left(Z^{\circ}\right) \rightarrow \pi_{1}(G)$ with $H^{2}(C, \mathbb{Z})=\mathbb{Z}$.

\section{Equivariant cohomology and partial localization}

In this section, let $V$ be a reduced, separated scheme of finite type over $\mathbb{C}$, viewed as a topological space in the analytic topology, with the action of a complex reductive algebraic group $\mathbb{G}_{m} \times G$.

\subsection{Fixed points on stacks}

The stack $V / G$ admits an action of the multiplicative group $\mathbb{G}_{m}$ induced from the product action on $V$; we write $(t, v) \mapsto t \cdot v$ for this action. Replacing that action with $a_{m}(t, v)=t^{m} \cdot v$ for each positive integer $m$, we obtain the action on $V / G$ of the pro-object $\widehat{\mathbb{G}}_{m}=\lim _{\leftarrow} \mathbb{G}_{m}$ in the category of algebraic groups, defined by all coverings of $\mathbb{G}_{m}$ by itself.

For simplicity write $\mathcal{X}=V / G$. When we wish to consider $\mathcal{X}$ with its $\mathbb{G}_{m}$-action via $a_{m}$, we write $\mathcal{X}_{a_{m}}$. Recall [Rom05, Definition 2.3] that a stack of fixed points $\mathcal{X}_{a_{m}}^{\mathbb{G}_{m}}$ is a stack $F$ that represents the 2 -functor $N \mapsto \operatorname{Hom}_{\mathbb{G}_{m} \text {-Stacks }}\left(\iota(N), \mathcal{X}_{a_{m}}\right)$, where $\iota$ takes a stack $N$ to the $\mathbb{G}_{m}$-stack with trivial $\mathbb{G}_{m}$-action. A fixed point $\operatorname{Spec}(\mathbb{C}) \rightarrow \mathcal{X}_{a_{m}}^{\mathbb{G}_{m}}$ can be viewed as a choice of section of $\left(\mathcal{X}_{a_{m}}\right) / \mathbb{G}_{m} \rightarrow B \mathbb{G}_{m}$.

There are morphisms $\mathcal{X}_{a_{m}}^{\mathbb{G}_{m}} \rightarrow \mathcal{X}_{a_{m} \cdot k}^{\mathbb{G}_{m}} \rightarrow \mathcal{X}$ for all $m, k>0$, defining $\mathcal{X}^{\widehat{\mathbb{G}}_{m}}:=\underline{\lim } \mathcal{X}_{a_{m}}^{\mathbb{G}_{m}} \longrightarrow \mathcal{X}$.

For each $x \in V$, write Lie $\left(\mathbb{G}_{m} \times G\right)_{x}$ for the Lie algebra of the stabilizer $\left(\mathbb{G}_{m} \times G\right)_{x}$. We write

$$
\left(V /\left(\mathbb{G}_{m} \times G\right)\right)_{\text {fixed }}=\left\{x \in V \mid \operatorname{Lie}\left(\mathbb{G}_{m} \times G\right)_{x} \rightarrow \operatorname{Lie}\left(\mathbb{G}_{m}\right) \text { is surjective }\right\} /\left(\mathbb{G}_{m} \times G\right) .
$$

Lemma 4.1. The condition above defines a closed substack $\left(V /\left(\mathbb{G}_{m} \times G\right)\right)_{\text {fixed }}$ of $V /\left(\mathbb{G}_{m} \times G\right)$. 


\section{On the KiRWAN MAP FOR MODUli OF Higgs BUndLES}

We write $\mathcal{X}_{\text {fixed }}=(V / G)_{\text {fixed }}$ for the preimage in $V / G$ of $\left(V /\left(\mathbb{G}_{m} \times G\right)\right)_{\text {fixed }}$ (cf. Appendix C of [GP99] in the case of DM stacks).

Proposition 4.2. The set of images of $\mathbb{C}$-points of $(V / G)^{\widehat{\mathbb{G}}_{m}} \rightarrow V / G$ is exactly $(V / G)_{\text {fixed }}$.

Proof. Suppose first that $x \in V$ and $\bar{x} \in(V / G)^{\widehat{\mathbb{G}}_{m}}$ have isomorphic images in $V / G$. Then there exists an $m>0$ such that $\bar{x}$ determines a section of $(V / G)_{a_{m}} / \mathbb{G}_{m} \rightarrow B \mathbb{G}_{m}$. The section induces homomorphisms $\mathbb{G}_{m} \rightarrow\left(\mathbb{G}_{m} \times G\right)_{x} \rightarrow \mathbb{G}_{m}$, where $\left(\mathbb{G}_{m} \times G\right)_{x}$ is defined with respect to the action of $\mathbb{G}_{m}$ via $a_{m}$, whose composite is the identity.

Conversely, suppose that $x \in V$ has image in $(V / G)_{\text {fixed }}$, so that $\operatorname{Lie}\left(\mathbb{G}_{m} \times G\right)_{x} \rightarrow \operatorname{Lie}\left(\mathbb{G}_{m}\right)$ is surjective. The stabilizer $\left(\mathbb{G}_{m} \times G\right)_{x}$ is the semidirect product of its unipotent radical and a Levi subgroup $L_{x}$, and the unipotent radical maps trivially to $\mathbb{G}_{m}$; it follows that the Levi subgroup $L_{x}$ of $\left(\mathbb{G}_{m} \times G\right)_{x}$ surjects onto $\mathbb{G}_{m}$. Since $L_{x} \rightarrow \mathbb{G}_{m}$ is a surjection of reductive complex groups, it can be split up to an isogeny of $\mathbb{G}_{m}$; choosing such a splitting, we get a homomorphism $\phi: \mathbb{G}_{m} \rightarrow\left(\mathbb{G}_{m} \times G\right)_{x}$ for which the composite $\mathbb{G}_{m} \stackrel{\phi}{\rightarrow} \mathbb{G}_{m} \times G \stackrel{\pi_{\mathbb{G}_{m}}}{\longrightarrow} \mathbb{G}_{m}$ is $\mathbb{G}_{m} \stackrel{()^{m}}{\longrightarrow} \mathbb{G}_{m}$ for some $m>0$. Taking quotients of $\operatorname{Spec}(\mathbb{C}) \stackrel{x}{\rightarrow} V \rightarrow \operatorname{Spec}(\mathbb{C})$ yields a section of $(V / G)_{a_{m}} / \mathbb{G}_{m}=$ $V /\left(\mathbb{G}_{m} \times G\right) \rightarrow B \mathbb{G}_{m}$.

Lemma 4.3. Assume that a subgroup $C \subset G$ acts trivially on $V$; let $r: V / G \rightarrow V /(G / C)$ be the quotient.

(1) A $\mathbb{C}$-point $x \in V / G$ lies in $(V / G)_{\text {fixed }}$ if and only if $r(x) \in(V /(G / C))_{\text {fixed }}$.

(2) In particular, if $V /(G / C)$ is a scheme, then $x \in(V / G)_{\text {fixed }}$ if and only if $r(x) \in(V /(G / C))^{\mathbb{G}_{m}}$.

Remark 4.4. Suppose $\mathcal{X}=V / G$ and that $X=V /(G / C)$ is a scheme. Then by the noetherianness of $X$ and Lemma 4.3, there is an $m>0$ for which $\mathcal{X}_{a_{m}}^{\mathbb{G}_{m}}(\mathbb{C}) \rightarrow X^{\mathbb{G}_{m}}(\mathbb{C})$ is already surjective.

Now, consider a reductive group $G$ acting on a smooth scheme $M$ with moment map $\mu$ : $T^{*} M \rightarrow \mathfrak{g}^{*}$, and let $V=\mu^{-1}(0)$. Let $\mathbb{G}_{m}$ act on $V \subset T^{*} M$ by fiberwise scaling, so $G \times \mathbb{G}_{m}$ acts on $V$. We denote points of $T^{*} M$ by $(x, \theta)$, where $x \in M$ and $\theta \in T_{x}^{*} M$.

Lemma 4.5. Suppose $V=\mu^{-1}(0) \subseteq T^{*} M$ as above. The $\mathbb{C}$-points of $(V / G)_{\text {fixed }}$ are the images of those $(x, \theta) \in V \subset T^{*} M$ such that there exist

(1) a homomorphism $\lambda: \mathbb{G}_{m} \rightarrow \operatorname{Stab}_{G}(x)$ and

(2) an $m>0$ such that $\lambda(t) \cdot(x, \theta)=\left(x, t^{m} \theta\right)\left(\right.$ for $\left.t \in \mathbb{G}_{m}\right)$ in $V$.

\subsection{A localization theorem for product actions}

As above, let $V$ be a reduced, separated scheme of finite type over $\mathbb{C}$, viewed as a topological space in the analytic topology, equipped with the action of a complex reductive algebraic group of the form $\mathbb{G}_{m} \times G$. Fix a maximal compact subgroup $K$ of $G$, so that $S^{1} \times K \subset \mathbb{G}_{m} \times G$ is a maximal compact subgroup. Consider the cohomology, always with $\mathbb{Q}$-coefficients, $H^{*}\left(\left(V /\left(\mathbb{G}_{m} \times G\right)\right)=\right.$ $H_{\mathbb{G}_{m} \times G}^{*}(V)=H_{S^{1} \times K}^{*}(V)$. We fix a generator $u \in H_{\mathbb{G}_{m}}^{2}(\mathrm{pt})$, so $H_{\mathbb{G}_{m}}^{*}(\mathrm{pt})=\mathbb{Q}[u]$. Write $\mathcal{Y}=$ $V /\left(\mathbb{G}_{m} \times G\right)$.

Proposition 4.6. The map $H^{*}(\mathcal{Y})\left[u^{-1}\right] \rightarrow H^{*}\left(\mathcal{Y}_{\text {fixed }}\right)\left[u^{-1}\right]$ is an isomorphism.

Proof. Write $(V / G)_{\text {fixed }}=F / G$ for the appropriate closed subset $F \subseteq V$. The proof mimics that of Theorem 4.2 of [Qui71]. By the Mayer-Vietoris argument of [Qui71, Theorem 4.2], it suffices to show that $H_{S^{1} \times K}^{*}(V \backslash F)\left[u^{-1}\right]=0$. Applying Remark 3.4 and formula (3.1) of [Qui71], it 


\section{E. Cliff, T. Nevins and S. Shen}

suffices to show that the image of $u$ in $H_{S^{1} \times K}^{*}\left(\left(S^{1} \times K\right) / H\right)$ is zero whenever $H \subset S^{1} \times K$ is a closed subgroup whose projection on $S^{1}$ is a proper (compact) subgroup of $S^{1}$.

Thus, assume that $H \subset S^{1} \times K$ is a closed subgroup whose image $\bar{H}$ in $S^{1}$ is finite. Then the $\operatorname{map} H_{S^{1}}^{*}(\mathrm{pt}) \rightarrow H_{S^{1} \times K}^{*}\left(\left(S^{1} \times K\right) / H\right)$ factors as

$$
H_{S^{1}}^{*}(\mathrm{pt}) \rightarrow H_{\bar{H}}^{*}(\mathrm{pt}) \rightarrow H_{H}^{*}(\mathrm{pt}) \cong H_{S^{1} \times K}^{*}\left(\left(S^{1} \times K\right) / H\right) .
$$

Since $\bar{H}$ is a finite group and we are working with $\mathbb{Q}$-coefficients, we have $H_{\bar{H}}^{2}(\mathrm{pt})=0$, and thus the image of $u$ in $H_{S^{1} \times K}^{*}\left(\left(S^{1} \times K\right) / H\right)$ vanishes, as required.

We explain a similar statement for intersection cohomology (always with $\mathbb{Q}$-coefficients), which will suffice for our purposes. As in the proof of Proposition 4.6, we restrict attention to the compact group $S^{1} \subset \mathbb{G}_{m}$. We continue to write $\mathcal{X}=V / G$. We abusively write $I H_{S^{1}}^{*}\left(\widehat{\mathcal{X}}_{\text {fixed }}\right)=$

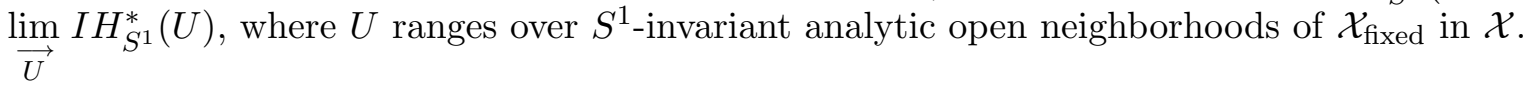

Proposition 4.7. The natural map $I H^{*}(\mathcal{X}) \rightarrow I H_{S^{1}}^{*}\left(\widehat{\mathcal{X}}_{\text {fixed }}\right)$ is an isomorphism.

The proof is a straightforward modification of the proof of Proposition 4.6, as in [Bry92, Corollary 4.1.3].

\section{Interlude: Regular nilpotent elements}

Recall the surjective homomorphism $\pi_{\mathrm{ad}}: G \rightarrow G_{\mathrm{ad}}$ that factors the adjoint homomorphism Ad: $G \rightarrow \mathrm{GL}(\mathfrak{g}), G_{\text {ad }}=\operatorname{Ad}(G)$. The group $G_{\text {ad }}$ is semisimple and $\operatorname{ker}\left(\pi_{\text {ad }}\right)=Z$. We have an isomorphism $[\mathfrak{g}, \mathfrak{g}] \stackrel{\cong}{\longrightarrow} \mathfrak{g}_{\mathrm{ad}}$, yielding a canonical $G$-equivariant splitting $\mathfrak{g}=\mathfrak{z} \oplus \mathfrak{g}_{\mathrm{ad}}$ as in Section 3.2.

Choose any regular nilpotent $e \in \mathfrak{g}_{\mathrm{ad}}$. It follows from the Jacobson-Morozov theorem that there exists a 1-parameter subgroup $\gamma: \mathbb{G}_{m} \rightarrow T \subseteq G$ with the property that $\operatorname{Ad}_{\gamma(t)}(e)=t^{m(\gamma)} e$ for some $m(\gamma)>0$, and that $\gamma$ can be chosen so that $m(\gamma)$ is either 1 or 2 .

Convention 5.1. We fix a $\gamma$ for which $m(\gamma)$ is (whichever of 1 and 2 is) the minimum possible.

The stabilizer $U_{\text {ad }}:=Z_{G_{\text {ad }}}(e)$ in $G_{\text {ad }}$ is a connected, commutative unipotent group [Spr66, Theorem 4.1]; it follows that $Z_{G}(e)=\pi_{\mathrm{ad}}^{-1}\left(Z_{G_{\mathrm{ad}}}(e)\right)$.

Lemma 5.2. There is a unique connected unipotent subgroup $\mathrm{U} \subset G$ for which $Z_{G}(e)=Z \times \mathrm{U}$.

Proof. Writing $\widetilde{G_{\text {ad }}}$ for the simply connected cover of $G_{\text {ad }}$, consider the commutative diagram

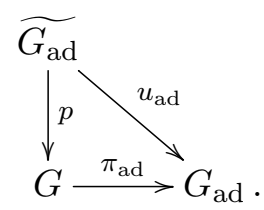

Let $\widetilde{U}:=u_{\mathrm{ad}}^{-1}\left(U_{\mathrm{ad}}\right)$ and $\mathrm{U}:=p(\widetilde{U})^{\circ}$. The homomorphism $\mathrm{U} \stackrel{\pi_{\mathrm{ad}}}{\longrightarrow} U_{\text {ad }}$ is surjective since its image has finite index by construction and $U_{\text {ad }}$ is connected; it has finite kernel since $u_{\text {ad }}$ has finite kernel. Since a unipotent group in characteristic zero has no nontrivial finite subgroups, we get that $\pi_{\text {ad }}$ induces an isomorphism $\mathrm{U} \cong U_{\text {ad }}$. Thus $\pi_{\text {ad }}^{-1}\left(U_{\text {ad }}\right)=Z \times U$. This proves the existence. For the uniqueness, note that if $U$ and $U^{\prime}$ are any two such subgroups, the composite 


\section{On the KiRWAN MAP FOR MODUli OF Higgs BUndLES}

$\mathrm{U}^{\prime} \subset Z_{G}(e)=Z \times \mathrm{U} \rightarrow Z$ is trivial because $Z$ is a product of a torus and a finite group. Thus $\mathrm{U}^{\prime} \subset \mathrm{U}$. Similarly, $\mathrm{U} \subset \mathrm{U}^{\prime}$, and we conclude $\mathrm{U}=\mathrm{U}^{\prime}$.

Corollary 5.3. (1) If $\operatorname{Ad}_{\gamma_{1}(t)}(e)=t^{m} e=\operatorname{Ad}_{\gamma_{2}(t)}(e)$, then there exists an $\eta: \mathbb{G}_{m} \rightarrow Z$ such that $\gamma_{2}=\eta \cdot \gamma_{1}$.

(2) For every 1-parameter subgroup $\lambda$ for which $\operatorname{Ad}_{\lambda(t)}(e)=t^{m(\lambda)}(e)$, there exist an $N>0$ and an $\eta: \mathbb{G}_{m} \rightarrow Z$ such that $\lambda(t)=\eta \cdot \gamma(t)^{N}$.

Proof. The product $\gamma_{2} \gamma_{1}^{-1}$ fixes $e$; hence it is a 1-parameter subgroup in $Z \times \mathrm{U}$, but $\mathrm{U}$ has no non-trivial 1-parameter subgroups.

The second assertion follows from the first together with the fact that the set of $N \in \mathbb{Z}$ for which there exists a 1-parameter subgroup $\lambda$ satisfying $\operatorname{Ad}_{\lambda(t)}(e)=t^{N} e$ is a cyclic subgroup in $\mathbb{Z}$.

Lemma 5.4. The $\operatorname{Ad}_{\gamma(t)}$-action on $\mathfrak{g}_{\text {ad }}$ induces a splitting $\mathfrak{g}_{\text {ad }}=\mathfrak{n}^{-} \oplus \mathfrak{t}_{\mathrm{ad}} \oplus \mathfrak{n}$, where $\mathfrak{t}_{\mathrm{ad}}$ is a Cartan subalgebra of $\mathfrak{g}_{\mathrm{ad}}$ and $\gamma(t)$ acts with positive weights on $\mathfrak{n}$ and negative weights on $\mathfrak{n}^{-}$.

It follows from [Hum95, Theorem 2.2] that for any integer $n>0$, the centralizer $Z_{G_{\text {ad }}}\left(\gamma^{n}\right)$ satisfies $Z_{G_{\text {ad }}}\left(\gamma^{n}\right)^{\circ}=T_{\text {ad }}$, where $T_{\text {ad }}=\pi_{\text {ad }}(T)$. Then by Lemma 5.2 above and Theorem 2.2 of [Hum95], we get the following.

Proposition 5.5. For any $n>0$, we have $Z\left(e, \gamma^{n}\right):=Z_{G}\left(\gamma^{n}\right) \cap Z_{G}(e)=Z$.

\section{Moduli of semistable Higgs bundles and fixed points}

We treat some properties of certain $\mathbb{G}_{m}$-fixed points on $\mathcal{M}_{\mathrm{Higgs}}^{\text {ss }}(G, C)$. A helpful discussion of the $\mathbb{G}_{m}$-action on the moduli space can be found in [GR15].

\subsection{Analysis of fixed points on $\mathcal{M}_{\text {Higgs }}^{\text {ss }}(G, C)$}

Suppose that $\left(P_{1}, \theta_{1}, \lambda_{1}\right)$ and $\left(P_{2}, \theta_{2}, \lambda_{2}\right)$ are points of $\mathcal{M}_{\text {Higgs }}^{\text {ss }}(G, C)_{\text {reg }}^{\widehat{\mathbb{G}}_{m}}$ (so $\theta_{1}$ and $\theta_{2}$ are regular in every fiber); that is, $P_{i}$ is a principal $G$-bundle, $\theta_{i}$ is a Higgs field on $P_{i}$, and $\lambda_{i}$ is a 1 parameter subgroup of $\operatorname{Aut}_{G}\left(P_{i}\right)$ that rescales $\theta_{i}$; that is, $\operatorname{Ad}_{\lambda_{i}(t)}\left(\theta_{i}\right)=t^{m_{i}} \theta_{i}$ for some integer $m_{i}$. This condition further implies that the Higgs fields $\theta_{1}$ and $\theta_{2}$ are regular nilpotent in every fiber. Replacing $\lambda_{1}$ and $\lambda_{2}$ with appropriate powers, we assume that $\theta_{i}$ is an eigenvector for $\lambda_{i}$ with the same exponent: $\operatorname{Ad}_{\lambda_{i}(t)}\left(\theta_{i}\right)=t^{N} \theta_{i}$.

Lemma 6.1. There exist a principal $Z$-bundle $P_{Z}$, a 1-parameter subgroup $\eta: \mathbb{G}_{m} \rightarrow Z$, and an isomorphism $\phi: P_{Z} \times{ }_{Z} P_{1} \stackrel{\cong}{\rightrightarrows} P_{2}$ of $G$-bundles such that $\phi\left(\theta_{1}\right)=\theta_{2}$ and $\phi\left(\eta \lambda_{1}\right)=\lambda_{2}$.

Proof. Choose a point $x \in C$. By Corollary 5.3, there exist a 1-parameter subgroup $\eta: \mathbb{G}_{m} \rightarrow Z$ and an isomorphism $\left(P_{1}\right)_{x} \cong\left(P_{2}\right)_{x}$ of principal homogeneous $G$-spaces that takes $\left(\theta_{1}\right)_{x}$ to $\left(\theta_{2}\right)_{x}$ and identifies $\eta \lambda_{1}$ with $\lambda_{2}$ on the fiber over $x$. Now, replacing $\lambda_{1}$ with $\eta \lambda_{1}$, we may assume that the fibers over $x$ of $\left(P_{1}, \theta_{1}, \lambda_{1}\right)$ and $\left(P_{2}, \theta_{2}, \lambda_{2}\right)$ are isomorphic. Proposition 5.5 implies that the bundle $P_{Z}:=\underline{\operatorname{Isom}}\left(\left(P_{1}, \theta_{1}, \lambda_{1}\right),\left(P_{2}, \theta_{2}, \lambda_{2}\right)\right)$ is a $Z$-torsor. Since $\operatorname{Hom}_{\mathrm{gp}}\left(\mathbb{G}_{m}, Z\right)$ is discrete, there exists an $\eta$ that guarantees $\phi\left(\eta \lambda_{1}\right)=\lambda_{2}$ on every fiber over $C$.

\subsection{Stable $G$-Higgs bundles with regular nilpotent Higgs field}

Lemma 6.2. Suppose that $(P, \theta)$ is a stable G-Higgs bundle with regular nilpotent Higgs field. Then $(P, \theta)$ is simple; that is, $\operatorname{Aut}(P, \theta)=Z$. 


\section{E. Cliff, T. Nevins and S. Shen}

Proof. By Lemma 5.2, the group scheme $\operatorname{Aut}(P, \theta)$ of fiberwise automorphisms of $(P, \theta)$ is the product of the constant group scheme $Z$ over $C$ and a unipotent group scheme $\mathrm{U}(P, \theta)$ over $C$. Because $(P, \theta)$ is stable, Proposition 3.15 of [GO17] implies that every global section of the automorphism group scheme, that is, element of $\operatorname{Aut}(P, \theta)$, is semisimple. The conclusion follows.

Proposition 6.3. Suppose that $(P, \theta)$ is any stable $G$-Higgs bundle with regular nilpotent Higgs field. Consider the morphism $a=a_{(P, \theta)}: \operatorname{Bun}(Z, C) \rightarrow \mathcal{M}_{\text {Higgs }}^{\text {st-spl }}(G, C)$ obtained by restricting the action morphism $\operatorname{Bun}(Z, C) \times \mathcal{M}_{\text {Higgs }}^{\text {ss }}(G, C) \rightarrow \mathcal{M}_{\text {Higgs }}^{\text {ss }}(G, C)$ to $\operatorname{Bun}(Z, C) \times\{(P, \theta)\}$. Then we obtain a commutative diagram

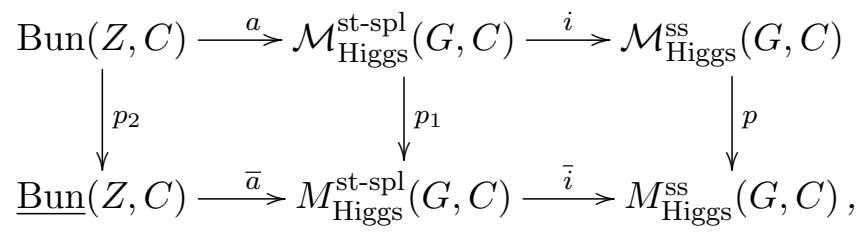

where

(1) the morphisms $p_{2}$ and $p_{1}$ are $B Z$-torsors, realizing $\operatorname{Bun}(Z, C)$ as the fiber product along $\bar{a}$ and $p_{1}$, and thus also realizing $\operatorname{Bun}(Z, C)$ as the fiber product along $\bar{i} \circ \bar{a}$ and $p$;

(2) the morphisms $\bar{i}$ and $i$ are open immersions; and

(3) the morphisms $\bar{a}, \bar{i} \circ \bar{a}, a$, and $i \circ a$ are closed immersions.

Proof. The existence of the commutative diagram is immediate from the constructions. Assertion (1) is immediate from the facts that $p_{2}$ and $p_{1}$ are both $B Z$-torsors and that $a$ is $B Z$ equivariant. Assertion (2) is immediate from Proposition 2.2(3). To prove assertion (3), we proceed as follows. By Proposition 3.3, each component of $\operatorname{Bun}(Z, C)$ is a projective variety; hence, the restrictions of $\bar{a}$ and $\bar{i} \circ \bar{a}$ to each component of $\operatorname{Bun}(Z, C)$ are proper. It follows from Proposition 3.8 that $\bar{a}$ and $\bar{i} \circ \bar{a}$ are of finite type; hence, $\bar{a}$ and $\bar{i} \circ \bar{a}$ are proper (the targets are separated, so properness lifts).

If we can show that $\bar{a}$ and $\bar{i} \circ \bar{a}$ are bijections onto their images on the level of $\mathbb{C}$-points, it will then follow from Corollary 3.6 that they separate tangents, hence are closed immersions. Thus, suppose that $\left(P_{1}, \theta_{1}\right)$ and $\left(P_{2}, \theta_{2}\right)$ are in the image of $\bar{a}$. As in the proof of Lemma 6.1, the automorphism group scheme $\operatorname{Aut}\left(P_{2}, \theta_{2}\right)$ is the product of the constant group scheme $Z$ over $C$ and a unipotent group scheme $\mathrm{U}(P, \theta)$ over $C$; similarly, it follows from Lemma 5.2 that the isomorphism bundle $\underline{\operatorname{Isom}}\left(\left(P_{1}, \theta_{1}\right),\left(P_{2}, \theta_{2}\right)\right)$ is an $\underline{\operatorname{Aut}}\left(P_{2}, \theta_{2}\right)$-torsor, which evidently is trivial if and only if $\left(P_{1}, \theta_{1}\right) \cong\left(P_{2}, \theta_{2}\right)$. We assume, without loss of generality in the proof, that $\left(P_{2}, \theta_{2}\right)=(P, \theta)$.

We consider the long exact sequence associated with $1 \rightarrow Z \times C \rightarrow \underline{\operatorname{Aut}}(P, \theta) \rightarrow \mathrm{U}(P, \theta) \rightarrow 1$,

$$
1 \rightarrow H^{0}(C, Z) \stackrel{F}{\rightarrow} H^{0}(C, \underline{\operatorname{Aut}}(P, \theta)) \rightarrow H^{0}(\mathrm{U}(P, \theta)) \rightarrow H^{1}(C, Z) \stackrel{\bar{a}}{\rightarrow} H^{1}(C, \underline{\operatorname{Aut}}(P, \theta)) \rightarrow \cdots .
$$

As noted above, $(P, \theta)$ is simple, and it follows that $F$ is an isomorphism. It follows from the "injectivity of tangent spaces" assertion of Corollary 3.6 that the fiber of $\bar{a}$ over $(P, \theta)$ is finite; hence, the image of the injective group homomorphism $H^{0}(\mathrm{U}(P, \theta)) \rightarrow H^{1}(C, Z)$ is finite, and in particular $H^{0}(\mathrm{U}(P, \theta))$ is finite. But the fibers of the group scheme $\mathrm{U}(P, \theta)$ are connected unipotent groups, which have no nontrivial finite subgroups, implying that $H^{0}(\mathrm{U}(P, \theta))=\{e\}$, and thus $\bar{a}$ is injective. Thus $\bar{a}$ and $\bar{i} \circ \bar{a}$ are indeed bijections onto their images, implying that they are closed immersions. 


\section{On the KiRWAN MAP FOR MODUli OF Higgs BUndLES}

It follows from assertion (1) and the previous paragraph that $a$ and $i \circ a$ are closed immersions.

\section{The main construction and the proof of Theorem 1.2}

\subsection{The main construction}

As in Section 5, assume that we have fixed (Convention 5.1) a 1-parameter subgroup $\gamma: \mathbb{G}_{m} \rightarrow G$ and a regular nilpotent $e \in \mathfrak{g}$ that is an eigenvector of $\operatorname{Ad}_{\gamma(t)}$ with eigenvalue $t^{m(\gamma)}$, where $m(\gamma)$ is either 1 or 2 .

DeFinition 7.1. We define

$$
P_{0}:=\mathrm{K}^{-1 / 2} \times_{\gamma_{1}} G, \quad \text { where } \quad \gamma_{1}(t)= \begin{cases}\gamma(t) & \text { if } m(\gamma)=2, \\ \gamma\left(t^{2}\right) & \text { if } m(\gamma)=1\end{cases}
$$

Thus $P_{0} \times_{G} \mathfrak{g}=\mathrm{K}^{-1 / 2} \times_{\gamma_{1}} \mathfrak{g}$. We let $\eta_{0}$ be the connected component of $\operatorname{Bun}(G, C)$ containing $P_{0}$.

Lemma 7.2. (1) The 1-parameter subgroup $\gamma$ determines a 1-parameter group $\lambda$ of automorphisms of $P_{0}$ by $\lambda(t) \cdot(a, p)=\left(a, \gamma_{1}(t) p\right)$ for $a \in \mathrm{K}^{1 / 2}, p \in G$.

(2) The element $e \in \mathfrak{g}$ determines a section $\theta_{0}$ of $\left(P_{0} \times_{G} \mathfrak{g}\right) \otimes \Omega_{C}^{1}$. Moreover, $\lambda(t)^{*} \theta_{0}=t^{2} \theta_{0}$.

Proof. Part (1) is clear by the construction of $P_{0}$. For part (2), note that

$$
\left(P_{0} \times_{G} \mathfrak{g}\right) \otimes \Omega_{C}^{1} \cong \mathrm{K}^{-1 / 2} \times_{\mathbb{G}_{m}}\left(\mathfrak{g} \otimes \mathbb{A}^{1}(-2)\right),
$$

where $\mathbb{G}_{m}$ acts on $\mathfrak{g}$ via $\gamma_{1}$. Since $e \in \mathfrak{g}$ has weight 2 with respect to $\gamma_{1}$, it determines an invariant element of $\mathfrak{g} \otimes \mathbb{A}^{1}(-2)$. Thus, $e$ descends to a section $\theta_{0}$, as asserted. That $\theta_{0}$ has weight 2 with respect to $\lambda$ is clear from the construction.

The Higgs pair $\left(P_{0}, \theta_{0}\right)$ is the image of the Hitchin section at 0 in the Hitchin base [Hit92, Section 5].

Lemma 7.3. The Higgs pair $\left(P_{0}, \theta_{0}\right)$ is stable.

Proof. By the Jacobson-Morozov theorem, $e$ lies in a unique Borel subalgebra $\mathfrak{b} \subset \mathfrak{g}$; thus any parabolic reduction of $P_{0}$ that is compatible with $\theta_{0}$ is of the form $\mathrm{K}^{-1 / 2} \times{ }_{\gamma_{1}} P$ for a parabolic $B \subseteq P \subseteq G$, where $B$ is the Borel subgroup corresponding to $\mathfrak{b}$. It now follows from Lemma 5.4 that the bundle $\mathrm{K}^{-1 / 2} \times_{\gamma_{1}} \mathfrak{g} / \mathfrak{p}$ has negative degree.

Applying Lemma 6.1, we conclude as follows.

Corollary 7.4. The pair $\left(P_{0}, \theta_{0}\right)$ defines a commutative diagram as in Proposition 6.3. The image of $a$ is exactly $\mathcal{M}_{\text {Higgs }}^{\text {ss }}(G, C)_{\text {fixed }} \cap \mathcal{M}_{\text {Higgs }}^{\text {ss }}(G, C)_{\text {reg }}$.

\subsection{Proof of Theorem 1.2}

That $H^{*}(\operatorname{Bun}(Z, C))$ acts trivially on $H^{*}(\operatorname{Bun}(G, C))$ is clear from the complex-analytic construction of $\operatorname{Bun}(G, C)$, which realizes the stack as a quotient of an (infinite-dimensional) affine space by a Lie group (again infinite-dimensional): the space $\operatorname{Bun}(Z, C)$ acts on the affine space of $\bar{\partial}$-operators; hence, the action is homotopically trivial. Thus, assertions (3) and (4) of the theorem are immediate from assertion (1).

From now on, we fix the pair $\left(P_{0}, \theta_{0}\right)$ of the main construction. By Corollary 7.4, the image of the corresponding morphism $\operatorname{Bun}(Z, C) \rightarrow \mathcal{M}_{\text {Higgs }}^{\text {ss }}(G, C)$ is $\mathcal{M}_{\text {Higgs }}^{\text {ss }}(G, C)_{\text {fixed }} \cap \mathcal{M}_{\text {Higgs }}^{\text {ss }}(G, C)_{\text {reg. }}$. 


\section{E. Cliff, T. Nevins and S. Shen}

The latter set is both open in $\mathcal{M}_{\text {Higgs }}^{\text {ss }}(G, C)_{\text {fixed }}$-because it is the intersection with the open subset of regular $G$-Higgs pairs - and closed because it is the image of a proper morphism. Thus, the image of $\operatorname{Bun}(Z, C) \rightarrow \mathcal{M}_{\text {Higgs }}^{\text {ss }}(G, C)$ consists of a union of connected components of $\mathcal{M}_{\text {Higgs }}^{\text {ss }}(G, C)_{\text {fixed }}$. The morphism $\operatorname{Bun}(Z, C) \rightarrow \mathcal{M}_{\text {Higgs }}^{\text {ss }}(G, C)$ being a closed immersion by Proposition 6.3, we conclude as follows.

Fact 7.5. The morphism $\operatorname{Bun}(Z, C) \rightarrow \mathcal{M}_{\mathrm{Higgs}}^{\mathrm{ss}}(G, C)$ determined by $\left(P_{0}, \theta_{0}\right)$ is a homeomorphism from $\operatorname{Bun}(Z, C)$ onto a union of connected components of $\mathcal{M}_{\text {Higgs }}^{\text {ss }}(G, C)_{\text {fixed }}$.

The finite abelian group stack $\operatorname{Bun}\left(\pi_{0}(Z), C\right)$-which is nontrivial by our main assumption that $\pi_{0}(Z)$ is nontrivial-acts on $\mathcal{M}_{\text {Higgs }}^{\text {ss }}(G, C)$, inducing compatible actions on $H^{*}\left(\mathcal{M}_{\text {Higgs }}^{\text {ss }}(G, C)\right)$ and $H^{*}\left(\mathcal{M}_{\text {Higgs }}^{\text {ss }}(G, C)_{\text {fixed }}\right)$. It follows from Proposition 3.7 that the action of $\operatorname{Bun}\left(\pi_{0}(Z), C\right)$ preserves connected components of $\operatorname{Bun}(G, C)$, hence by [GO17] preserves connected components of $\mathcal{M}_{\text {Higgs }}^{\text {ss }}(G, C)$.

Consider the Leray spectral sequence for $\mathbb{G}_{m}$-equivariant cohomology,

$$
E_{2}^{*, *}=H^{*}\left(\mathcal{M}_{\mathrm{Higgs}}^{\mathrm{ss}}(G, C), \mathbb{Q}\right) \otimes H_{\mathbb{G}_{m}}^{*}(\mathrm{pt}, \mathbb{Q}) \Longrightarrow H_{\mathbb{G}_{m}}^{*}\left(\mathcal{M}_{\mathrm{Higgs}}^{\mathrm{ss}}(G, C), \mathbb{Q}\right) .
$$

Writing $H_{\mathbb{G}_{m}}^{*}(\mathrm{pt}, \mathbb{Q}) \cong \mathbb{Q}[u]$ and localizing to $\mathbb{Q}\left[u^{ \pm 1}\right]$ transforms the spectral sequence into

$$
E_{2}^{*, *}=H^{*}\left(\mathcal{M}_{\mathrm{Higgs}}^{\mathrm{ss}}(G, C), \mathbb{Q}\right) \otimes H_{\mathbb{G}_{m}}^{*}(\mathrm{pt}, \mathbb{Q})\left[u^{-1}\right] \Longrightarrow H_{\mathbb{G}_{m}}^{*}\left(\mathcal{M}_{\mathrm{Higgs}}^{\mathrm{ss}}(G, C), \mathbb{Q}\right)\left[u^{-1}\right] .
$$

Remark 7.6. We note that the mixed Hodge structure on $H_{\mathbb{G}_{m}}^{*}(\mathrm{pt}, \mathbb{Q})\left[u^{-1}\right]$ is pure; thus, only the pure part of $H^{*}\left(\mathcal{M}_{\mathrm{Higgs}}^{\mathrm{ss}}(G, C), \mathbb{Q}\right)$ contributes to the pure part of $H_{\mathbb{G}_{m}}^{*}\left(\mathcal{M}_{\mathrm{Higgs}}^{\mathrm{ss}}(G, C), \mathbb{Q}\right)\left[u^{-1}\right]$.

Applying Propositions 4.6 and 4.2, we know that the spectral sequence abuts to

$$
H_{\mathbb{G}_{m}}^{*}\left(\mathcal{M}_{\text {Higgs }}^{\mathrm{ss}}(G, C)_{\text {fixed }}, \mathbb{Q}\right)\left[u^{-1}\right] .
$$

The spectral sequence is equivariant with respect to all $\mathbb{G}_{m}$-compatible automorphisms of $\mathcal{M}_{\text {Higgs }}^{\text {ss }}(G, C)$, in particular with respect to the $\operatorname{Bun}\left(\pi_{0}(Z), C\right)$-action.

Lemma 7.7. The graded representation $H_{\mathbb{G}_{m}}^{*}\left(\mathcal{M}_{\text {Higgs }}^{\mathrm{ss}}(G, C)_{\text {fixed }}, \mathbb{Q}\right)\left[u^{-1}\right]$ of $\operatorname{Bun}\left(\pi_{0}(Z), C\right)$ contains a copy of $\mathbb{Q}\left[\operatorname{Bun}\left(\pi_{0}(Z), C\right)\right]$ lying in graded degree 0 (and consisting of pure classes for the mixed Hodge structure).

Proof. The group $\operatorname{Bun}\left(\pi_{0}(Z), C\right)$ acts freely on $\pi_{0}(\operatorname{Bun}(Z, C))$; hence, $H^{*}(\operatorname{Bun}(Z, C)$, $\mathbb{Q})$ contains a copy of the regular representation of $\operatorname{Bun}\left(\pi_{0}(Z), C\right)$ in degree 0 , implying that

$$
H_{\mathbb{G}_{m}}^{*}(\operatorname{Bun}(Z, C), \mathbb{Q})=H^{*}(\operatorname{Bun}(Z, C), \mathbb{Q}) \otimes H_{\mathbb{G}_{m}}^{*}(\mathrm{pt})
$$

does as well (here $\mathbb{G}_{m}$ acts trivially on $\operatorname{Bun}(Z, C)$ ); hence by Fact 7.5, the same assertion is true of $H_{\mathbb{G}_{m}}^{*}\left(\mathcal{M}_{\mathrm{Higgs}}^{\mathrm{ss}}(G, C)_{\mathrm{fixed}}, \mathbb{Q}\right)\left[u^{-1}\right]$.

We continue with the proof of Theorem 1.2. Since $H_{\mathbb{G}_{m}}^{*}\left(\mathcal{M}_{\mathrm{Higgs}}^{\mathrm{ss}}(G, C)_{\text {fixed }}, \mathbb{Q}\right)\left[u^{-1}\right]$ can be obtained by taking iterated $\operatorname{Bun}\left(\pi_{0}(Z), C\right)$-equivariant subquotients of $H^{*}\left(\mathcal{M}_{\text {Higgs }}^{\text {ss }}(G, C), \mathbb{Q}\right) \otimes$ $H_{\mathbb{G}_{m}}^{*}(\mathrm{pt}, \mathbb{Q})\left[u^{-1}\right]$, it then follows that the latter contains a copy of $\mathbb{Q}\left[\operatorname{Bun}\left(\pi_{0}(Z), C\right)\right]$ lying in graded degree 0 . Finally, note that for any graded vector space $V^{*}$, the natural map $V^{*} \rightarrow$ $V^{*}\left[u^{ \pm 1}\right]_{0}$ acting by multiplication by $u^{-k}$ on $V^{k}$ is an isomorphism. It then follows from this observation and Lemma 7.7 that $H^{*}\left(\mathcal{M}_{\mathrm{Higgs}}^{\mathrm{ss}}(G, C), \mathbb{Q}\right)$ contains a copy of $\mathbb{Q}\left[\operatorname{Bun}\left(\pi_{0}(Z), C\right)\right]$, as desired. 


\section{On the KiRWAN MAP FOR MOdUli OF HigGS BUndles}

It remains to prove a similar assertion for the moduli space $M_{\mathrm{Higgs}}^{\mathrm{ss}}(G, C)$. It follows from Propositions 6.3 and 2.2(iii), Lemma 4.3(ii), and Fact 7.5 that $\underline{\operatorname{Bun}}(Z, C) \rightarrow M_{\mathrm{Higgs}}^{\mathrm{ss}}(G, C)^{\mathbb{G}_{m}}$ is a homeomorphism onto a union of connected components of $M_{\text {Higgs }}^{\text {ss }}(G, C)^{\mathbb{G}_{m}}$. Since $\pi_{0}(\operatorname{Bun}(Z, C))=$ $\pi_{0}(\underline{\operatorname{Bun}}(Z, C))$, we conclude that $\operatorname{Bun}\left(\pi_{0}(Z), C\right)$ again acts nontrivially on $H^{*}\left(M_{\text {Higgs }}^{\text {ss }}(G, C), \mathbb{Q}\right)$. Further, it follows from the functoriality of the Leray spectral sequence that the homomorphism $H^{*}\left(M_{\text {Higgs }}^{\text {ss }}(G, C), \mathbb{Q}\right) \rightarrow H^{*}\left(\mathcal{M}_{\text {Higgs }}^{\text {ss }}(G, C), \mathbb{Q}\right)$ must take some nontrivial $\operatorname{Bun}\left(\pi_{0}(Z), C\right)$-isotypic components of the domain (corresponding to part of $H^{0}\left(M_{\mathrm{Higgs}}^{\mathrm{ss}}(G, C)^{\mathbb{G}_{m}}\right)$ ) isomorphically onto their images. This completes the proof of assertion (1).

The intersection cohomology assertions (2) follow from the same proof applied to $I H^{*}$ rather than $H^{*}$ : the crucial observation is that the union of connected components given by the image of $\operatorname{Bun}(Z, C)$ lies in the rationally smooth locus of $\mathcal{M}_{\text {Higgs }}^{\text {ss }}(G, C)$, where the colimit appearing in the target of Proposition 4.7 reduces to the singular cohomology of the (smooth) fixed stack $\left(\mathcal{M}_{\text {Higgs }}^{\text {ss }}(G, C)\right)_{\text {fixed. }}$ This completes the proof.

Remark 7.8. In light of Remark 7.6, the proof shows that the representation $\mathbb{Q}\left[\operatorname{Bun}\left(\pi_{0}(Z), C\right)\right]$ appears in the pure part of the mixed Hodge structures on the cohomology $H^{*}\left(\mathcal{M}_{\text {Higgs }}^{\text {ss }}(G, C), \mathbb{Q}\right)$ and $H^{*}\left(M_{\text {Higgs }}^{\text {ss }}(G, C), \mathbb{Q}\right)$.

\section{ACKNOWLEDGEMENTS}

We are grateful to Kevin McGerty for many conversations, and to Brian Collier, Kevin McGerty, Steven Rayan, Charles Rezk, Laura Schaposnik, and Eric Vasserot for help with references.

\section{REFERENCES}

Alp13 J. Alper, Good moduli spaces for Artin stacks, Ann. Inst. Fourier (Grenoble) 63 (2013), no. 6, 2349-2402; doi:10.5802/aif. 2833.

Bry92 J.-L. Brylinski, Equivariant intersection cohomology, Kazhdan-Lusztig Theory and Related Topics (Chicago, IL, 1989), Contemp. Math., vol. 139 (Amer. Math. Soc., Providence, RI, 1992), 5-32; doi:10.1090/conm/139/1197827.

DWWW11 G. Daskalopoulos, J. Weitsman, R. A. Wentworth, and G. Wilkin, Morse theory and hyperkähler Kirwan surjectivity for Higgs bundles, J. Differential Geom. 87 (2011), no. 1, 81115; doi:10.4310/jdg/1303219773.

GO17 O. García-Prada and A. Oliveira, Connectedness of Higgs bundle moduli for complex reductive Lie groups, Asian J. Math. 21 (2017), no. 5, 791-810; doi:10.4310/A JM. 2017.v21.n5.a1.

GP99 T. Graber and R. Pandharipande, Localization of virtual classes, Invent. Math. 135 (1999), no. 2, 487-518; doi:10.1007/s002220050293.

GR15 A. García-Raboso and S. Rayan, Introduction to nonabelian Hodge theory: flat connections, Higgs bundles and complex variations of Hodge structure, in Calabi-Yau Varieties: Arithmetic, Geometry and Physics, Fields Inst. Monogr., vol. 34 (Fields Inst. Res. Math. Sci., Toronto, ON, 2015), 131-171; doi:10.1007/978-1-4939-2830-9_5.

Hit87 N. J. Hitchin, The self-duality equations on a Riemann surface, Proc. London Math. Soc. 55 (1987), no. 1, 59-126; doi:10.1112/plms/s3-55.1.59.

Hit92 Lie groups and Teichmüller space, Topology 31 (1992), no. 3, 449-473; doi:10. 1016/0040-9383(92) 90044-I.

Hof10 N. Hoffmann, On moduli stacks of G-bundles over a curve, in Affine Flag Manifolds and Principal Bundles, Trends Math. (Birkhäuser/Springer Basel AG, Basel, 2010), 155-163; doi:10.1007/978-3-0346-0288-4_5. 


\section{E. Cliff, T. Nevins and S. Shen}

HT03 T. Hausel and M. Thaddeus, Mirror symmetry, Langlands duality, and the Hitchin system, Invent. Math. 153 (2003), no. 1, 197-229; doi:10.1007/s00222-003-0286-7.

Hum95 J.E. Humphreys, Conjugacy classes in semisimple algebraic groups, Math. Surveys Monogr., vol. 43 (Amer. Math. Soc., Providence, RI, 1995); doi:10.1090/surv/043.

JKK09 L. Jeffrey, Y.-H. Kiem, and F. Kirwan, On the cohomology of hyperkähler quotients, Transform. Groups 14 (2009), no. 4, 801-823; doi:10.1007/s00031-009-9065-x.

Kir84 F. C. Kirwan, Cohomology of quotients in symplectic and algebraic geometry, Math. Notes, vol. 31 (Princeton Univ. Press, Princeton, NJ, 1984); doi:10.1007/BF01145470.

KW07 A. Kapustin and E. Witten, Electric-magnetic duality and the geometric Langlands program, Commun. Number Theory Phys. 1 (2007), no. 1, 1-236; doi:10.4310/CNTP.2007.v1.n1.a1.

Mar02 E. Markman, Generators of the cohomology ring of moduli spaces of sheaves on symplectic surfaces, J. reine angew. Math. 544 (2002), 61-82; doi:10.1515/crll.2002.028.

MN18 K. McGerty and T. Nevins, Kirwan surjectivity for quiver varieties, Invent. Math. 212 (2018), no. 1, 161-187; doi:10.1007/s00222-017-0765-x.

MN19_ Counterexamples to hyperkähler Kirwan surjectivity, 2019, arXiv:1904.12003.

Qui71 D. Quillen, The spectrum of an equivariant cohomology ring. I, Ann. of Math. 94 (1971), no. 3, 549-572; doi:10.2307/1970770.

Rom05 M. Romagny, Group actions on stacks and applications, Michigan Math. J. 53 (2005), no. 1, 209-236; doi:10.1307/mmj/1114021093.

Spr66 T. A. Springer, Some arithmetical results on semi-simple Lie algebras, Publ. Math. Inst. Hautes Études Sci. 30 (1966), 115-141; doi:10.1007/BF02684358.

Emily Cliff emily.cliff@sydney.edu.au

Sydney Mathematical Research Institute, University of Sydney, The Quadrangle, Camperdown NSW 2006, Australia

Thomas Nevins

Department of Mathematics, University of Illinois at Urbana-Champaign, Urbana, IL 61801, USA

Shiyu Shen shiyu.shen@utoronto.ca

Department of Mathematics, University of Toronto, Bahen Centre, 40 St. George St., Toronto, Ontario M5S 2E4, Canada 